\title{
TEORES DE CORANTES ARTIFICIAIS EM ALIMENTOS DETERMINADOS POR CROMATOGRAFIA LÍQUIDA DE ALTA EFICIÊNCIA
}

\author{
Marcelo Alexandre Prado* e Helena Teixeira Godoy \\ Departamento de Ciência de Alimentos, Faculdade de Engenharia de Alimentos, Universidade Estadual de Campinas, CP 6121, \\ 13083-862 Campinas - SP, Brasil \\ Recebido em 14/7/05; aceito em 25/5/06; publicado na web em 26/9/06
}

\begin{abstract}
CONTENTS OF SYNTHETIC DYES IN FOODS DETERMINED BY HIGH PERFORMENCE LIQUID CHROMATOGRAPHY. Synthetic dyes are much used in processed foods. HPLC was applied to different types of snacks, such as colored cereals, chocolate confetti, chewing gums and candies for the determination of those additives. In the case of artificially colored breakfast cereals, $71 \%$ of the samples exceeded the allowed limits. Regarding the portions recommended for consumption by the makers of two of the samples, the amounts exceeded those allowed by the Brazilian legislation. In the case of chocolate confetti and candies none of the samples showed higher amounts than those allowed. However $37 \%$ of the chewing gum samples presented larger contents than the authorized ones, and one sample contained five times more synthetic dyes than allowed.
\end{abstract}

Keywords: synthetic dyes; HPLC; foods.

\section{INTRODUÇÃO}

Enquanto a maioria das empresas do setor de alimentação sofre com a crise econômica, as indústrias de balas, bombons, dropes e gomas de mascar nem chegaram a sentir os efeitos da crise. Ao contrário, fabricar e vender guloseimas tem engordado, ano após ano, o faturamento de mais de 100 empresas do ramo ${ }^{1}$.

Com produtos baratos, como as balas avulsas que podem custar apenas alguns centavos, os fabricantes de guloseimas colocam em prática o velho ditado popular, reunindo milhões de tostão em tostão. Também no varejo, trata-se de uma linha lucrativa e indispensável para os mais de 400 mil revendedores espalhados por todo o país. O consumo de guloseimas, praticamente, não pesa no bolso, e uma bala muitas vezes substitui a moeda do troco. A característica preço, aliada à farta rede de vendas, garante o sucesso do setor. Uma vez que o setor é extremamente competitivo, oferecendo 220 marcas diferentes para uma linha, que não ultrapassa 40 tipos de produtos, as empresas lançam mão de diversos artifícios para conquistar o mercado, desde altos investimentos em propaganda, até tornar as guloseimas as mais atrativas possíveis, o que justifica a grande utilização de corantes artificiais para tal finalidade ${ }^{1}$.

O mesmo vem ocorrendo com outros produtos, como bebidas não alcoólicas e cereais matinais coloridos. Infelizmente, o consumidor por si só não é capaz de controlar a própria exposição aos corantes e, consequientemente, não pode avaliar se o benefício recebido justifica o risco a que está exposto ${ }^{2,3}$.

A manutenção da cor natural do alimento constitui-se em um fator fundamental para o marketing do produto, em face da primeira avaliação do consumidor. Antes do paladar, os alimentos coloridos seduzem as pessoas pela visão. A lógica do consumo desses produtos inicia-se pelos olhos: alimentos coloridos, vistosos, atraentes só podem ser deliciosos. Em geral, a importância da aparência do produto para sua aceitabilidade é a maior justificativa para o emprego de corantes ${ }^{4}$.

Os estudos sobre os efeitos à saúde causados pelos corantes artificiais são insuficientes e bastante contraditórios. Diversos autores relatam as reações adversas que podem causar, como reações

*e-mail:mprado@fea.unicamp.br alérgicas $^{5,6}$, enquanto outros demonstram a ação antitumoral de alguns deles ${ }^{7}$.

Os aditivos são inofensivos à saúde, desde que se obedeça aos percentuais máximos estabelecidos pela ANVISA (Agencia Nacional de Vigilância Sanitária) e/ou pelo Codex Alimentarius. Estes estabelecem para cada aditivo uma ingestão diária aceitável (IDA). Todos os corantes artificiais permitidos pela Legislação Brasileira já possuem valor definido de IDA, embora esses valores estejam sujeitos a alterações contínuas, dependendo dos resultados de estudos toxicológicos. O Comitê conjunto FAO/OMS de peritos em aditivos alimentares, JECFA ("Joint Expert Committee on Food Additives"), em nível internacional, recomenda que cada país verifique periodicamente o consumo total de cada aditivo, com base em estudos de dieta, para se assegurar que a ingestão total do aditivo não ultrapasse a IDA ${ }^{8,9}$.

\section{CORANTES ARTIFICIAIS PERMITIDOS NO BRASIL}

É permitido no Brasil o uso de onze corantes artificiais, sendo eles Tartrazina (E-102), Amarelo Crepúsculo (E-110), Azorrubina (E-122), Amaranto (E123), Ponceau 4R (E-124), Eritrosina (E-127), Vermelho 40 (E-129), Azul Patente V (E-131), Indigotina (E-132), Azul Brilhante (E-133) e Verde Rápido (E143) ${ }^{10}$.

Neste trabalho foram utilizados oito dos onze corantes artificiais permitidos, sendo eles E-102, E-110, E123, E-124, E-127, E129, E-132, E-133 ${ }^{10}$.

A regulamentação dos corantes e a necessidade de controle de qualidade do governo sobre as empresas têm forçado o desenvolvimento de novas técnicas analíticas capazes de responder não só à demanda do número de análises mas, de forma rápida e, principalmente, confiável. Com essa finalidade é que, atualmente, vários métodos envolvendo a cromatografia líquida de alta eficiência (CLAE) estão sendo usados para determinar qualitativa e quantitativamente esse tipo de aditivo ${ }^{11}$.

O presente trabalho teve como objetivo a determinação simultânea dos corantes permitidos no Brasil em vários tipos de alimentos, através de uma metodologia utilizando a CLAE ${ }^{2,3}$. A escolha de guloseimas e cereais matinais coloridos para as análises deve-se, principalmente, ao fato de serem produtos amplamente consumi- 
dos, especialmente pela população infantil, e por apresentarem corantes artificiais em sua composição.

\section{PARTE EXPERIMENTAL}

\section{Material}

Foram analisados alguns tipos de guloseimas de vários sabores, sendo balas, gomas de mascar e confeitos de chocolate coloridos artificialmente e mais três marcas diferentes de cereais matinais, que contêm vários sabores. Para todas as amostras foram analisados 2 lotes diferentes, identificados pela data de fabricação. As amostras foram preparadas separando-se por cores/sabores. As análises foram sempre realizadas em duplicata. Antes da tomada da amostra analítica, os alimentos foram homogeneizados. Todos os produtos foram comprados em supermercados da região de Campinas, SP.

\section{Reagentes}

Os padrões de corantes artificiais foram adquiridos da Importadora Brastóquio ltda. O metanol, utilizado com grau cromatográfico "ominsolv", e o acetato de amônio foram adquiridos da Merck Brasil. A água utilizada no preparo das fases móveis e das amostras foi purificada no sistema Milli-Q (Millipore). As fases móveis foram sempre filtradas em filtros Floupore (Millipore HAWP 0013) de 0,5 $\mu \mathrm{m}$ de diâmetro de poro, e degaseificadas em banho ultra-sônico.

\section{Equipamento}

Para análise foi utilizado cromatógrafo a líquido HP série 1050, com sistema de bombeamento isocrático; válvula injetora tipo "Rheodyne", com alça de amostragem de $20 \mu \mathrm{L}$ e detector de arranjo de diodos (DAD) HP série 1050, acoplado a um software HP Chemstation, que apresenta o recurso de visualização de até cinco comprimentos de onda diferentes em uma mesma corrida. Foram utilizados os comprimentos de onda de $595 \mathrm{~nm}$ para os azuis, 525 $\mathrm{nm}$ para os vermelhos e $450 \mathrm{~nm}$ para os amarelos.

Para separação cromatográfica utilizou-se uma coluna Spherisorb ODS-2 de $150 \mathrm{~mm}$ x 4,6 mm d. i., $\mathrm{C}_{18}$ de $5 \mu \mathrm{m}$, protegida por uma coluna de guarda Micropore $30 \mathrm{~mm}$ x 4,6 mm d.i. $\mathrm{C}_{18}$, de $10 \mu \mathrm{m}$.

\section{Métodos analíticos}

Pesou-se entre 4 a $9 \mathrm{~g}$ de balas, gomas e confeitos de chocolate coloridos (em geral eram tomadas algumas unidades de cada amostra), para a extração dos corantes. As amostras foram simplesmente dissolvidas em água quente $\left(40\right.$ a $\left.50{ }^{\circ} \mathrm{C}\right)$, e o volume ajustado para $50 \mathrm{~mL}$. Em especial, os confeitos de chocolate e gomas foram lavados várias vezes com água, para garantir a completa extração.

Para os cereais matinais coloridos pesou-se entre 3 a $5 \mathrm{~g}$ de amostra, previamente homogeneizada. As análises foram realizadas separando-se as amostras por cores/sabores e na porção recomendada para consumo pelo fabricante $(30 \mathrm{~g})$. Solução amoniacal $10 \%$ em etanol mostrou-se mais eficiente que a água para a extração dos corantes em cereais matinais coloridos. Lavagens sucessivas foram realizadas, até a completa retirada dos corantes. As alíquotas retiradas eram unidas e concentradas em roto evaporador $\left(70{ }^{\circ} \mathrm{C}\right)$ até um volume final de $50 \mathrm{~mL}$. As amostras tiveram seus volumes ajustados com água, obtida do sistema de filtração Milli Q. Os volumes finais das amostras, de porção recomendada, tiveram seus volumes finais ajustados para $250 \mathrm{~mL}$, por se tratar de uma quantidade inicial 10 vezes maior que a normalmente utilizada.
Em todas as amostras, após o ajuste de volume, era coletada uma fração, e centrifugada a uma rotação de $15.000 \mathrm{rpm}$ por $10 \mathrm{~min}$.

Após a centrifugação, o sobrenadante (cerca de $10 \mathrm{~mL}$ ) era filtrado em membrana tipo HAWP de $0,5 \mu \mathrm{m}$ (Millipore), e injetado no cromatógrafo.

Todas as amostras foram analisadas em duplicata, exceto a porção recomendada de cereais matinais coloridos que foi realizada em quadriplicata, coletando-se a amostragem aleatoriamente do pacote.

\section{Cromatografia}

Para análise em CLAE foi utilizado um cromatógrafo a líquido HP (Helwett Packard) série 1050, com sistema de bombeamento isocrático e válvula injetora tipo "Rheodyne" e uma alça de amostragem de $20 \mu \mathrm{L}$ de capacidade. Para separação dos compostos foi utilizada uma coluna cromatográfica Spherisorb ODS-2, com partículas de $5 \mu \mathrm{m}$, com dimensões de 150 x 4,6 mm d.i. (SigmaAldrich, USA) protegida por uma coluna de guarda Micropore, $\mathrm{C}_{18}, 10 \mu \mathrm{m}, 30 \mathrm{x}$ 4,6 mm d.i. (Varian). Um detector de arranjo de diodos (DAD) da HP série 1050, acoplado a um software HP Chemstation, permitiu registrar o cromatograma em três diferentes comprimentos de onda, durante uma mesma corrida, o que possibilitou a análise de todos os corantes de forma simultânea.

Os corantes foram separados através de um sistema de eluição isocrático. A coluna era condicionada pela passagem de uma solução de água/metanol 70:30 + 0,08 M de acetato de amônio por 12,5 min e, após esse período, era acionada a corrida com mudança de fase móvel para água/metanol 70:30. Em ambos os casos a vazão era de $0,5 \mathrm{~mL} / \mathrm{min}$. O tempo de corrida para a separação dos corantes era de $20 \mathrm{~min}^{2,3}$.

\section{Detecção e identificação}

Os picos foram detectados através do detector de arranjo de diodo (DAD). Os corantes foram divididos em três grupos: os azuis foram detectados a $595 \mathrm{~nm}$, os vermelhos a $525 \mathrm{~nm}$ e os amarelos a $450 \mathrm{~nm}$.

Os corantes foram identificados pelos tempos de retenção em comparação com os tempos de retenção dos padrões analisados nas mesmas condições e pelos espectros de absorção obtidos pelo DAD. A adição de padrões nas amostras também foi usada como parâmetro de identificação (co-cromatografia). A quantificação foi realizada por padronização externa. Foram construídas curvas de padronização para cada corante artificial, com 5 níveis de concentração, sendo cada ponto a média de duas injeções.

\section{RESULTADOS E DISCUSSÃO}

As composições qualitativas e quantitativas dos corantes artificiais presentes nas amostras são apresentadas nas Tabelas 1 a 4 . Os limites permitidos pela legislação brasileira são: Azorrubina (E-122) e Eritrosina (E-127) 5 mg/100 g; Amarelo Crepúsculo (E-110), Amaranto (E123) e Ponceau 4R (E-124) 10 mg/100 g; Tartrazina (E102), Vermelho 40 (E-129), Azul Patente V (E-131), Indigotina (E132), Azul Brilhante (E-133) e Verde Rápido (E143) 30 mg/100 g.

Em confeitos de chocolate e balas nenhuma das amostras apresentou índices superiores aos permitidos.

Nos confeitos de chocolate coloridos, todos os teores estavam dentro do permitido (Tabela 1), e em duas amostras cor/sabor marrom das marcas B e D, foi encontrada mistura de 4 corantes.

Nos produtos de goma de mascar, 9 das 27 amostras analisadas (33\% do total) foram encontrados valores maiores que os autorizados para esse tipo de produto e no sabor limão do fabricante A foi encontrado cinco vezes mais corante que o permitido (Tabela 2). 
Tabela 1. Teores de corantes artificiais encontrados em confeitos de chocolate coloridos*

\begin{tabular}{|c|c|c|c|c|c|c|c|c|c|c|}
\hline MARCA & $\begin{array}{c}\text { COR/ } \\
\text { SABOR }\end{array}$ & E-102 & E-110 & $\begin{array}{c}\text { CORANTES } \\
\text { E-123 }\end{array}$ & E-124 & $\begin{array}{c}(\mathrm{mg} / 100 \mathrm{~g}) \\
\mathrm{E}-127\end{array}$ & E-129 & E-132 & E-133 & TOTAL \\
\hline \multirow[t]{7}{*}{ A } & azul & & & & & & & & $0,87 \pm 0,00$ & 0,87 \\
\hline & amarelo & $1,84 \pm 0,02$ & & & & & & & & 1,84 \\
\hline & vermelho & & & $7,0 \pm 0,3$ & $4,03 \pm 0,08$ & & $2,24 \pm 0,03$ & & & 13,27 \\
\hline & verde & $2,95 \pm 0,04$ & & & & & & & $0,75 \pm 0,00$ & 3,70 \\
\hline & rosa & & & & & $1,64 \pm 0,01$ & & & & 1,64 \\
\hline & laranja & & $5,2 \pm 0,1$ & & Traços & & & & & 5,24 \\
\hline & marrom & & $5,5 \pm 0,2$ & & & & $1,57 \pm 0,01$ & $0,58 \pm 0,00$ & & 7,64 \\
\hline \multirow[t]{5}{*}{ B } & azul & & & & & & & $0,64 \pm 0,00$ & & 0,64 \\
\hline & laranja & & $6,1 \pm 0,2$ & & & & & & & 6,11 \\
\hline & vermelho & & & $5,9 \pm 0,2$ & $3,37 \pm 0,06$ & & & & & 9,23 \\
\hline & amarelo & $4,5 \pm 0,1$ & & & & & & & & 4,52 \\
\hline & marrom & & $7,5 \pm 0,3$ & $1,26 \pm 0,01$ & $0,72 \pm 0,00$ & & & $0,77 \pm 0,00$ & & 10,21 \\
\hline \multirow[t]{8}{*}{$\mathrm{C}$} & azul & & & & & & & & $1,29 \pm 0,01$ & 1,29 \\
\hline & amarelo & $2,80 \pm 0,04$ & traços & & & & & & & 2,80 \\
\hline & verde & $1,68 \pm 0,01$ & & & & & & & $0,54 \pm 0,00$ & 2,22 \\
\hline & rosa & & & & & traços & & & & \\
\hline & vermelho & & & $1,25 \pm 0,05$ & $1,80 \pm 0,02$ & & & & & 3,05 \\
\hline & laranja & & $4,6 \pm 0,1$ & & & & & & & 4,59 \\
\hline & roxo & & traços & $1,25 \pm 0,01$ & & & & $0,30 \pm 0,00$ & & 1,55 \\
\hline & marrom & $2,25 \pm 0,03$ & & & $0,87 \pm 0,00$ & & & $0,30 \pm 0,00$ & & 3,42 \\
\hline \multirow[t]{5}{*}{ D } & verde & $3,54 \pm 0,06$ & & & & & & & $1,10 \pm 0,00$ & 4,64 \\
\hline & azul & & & & & & & $1,82 \pm 0,01$ & $2,81 \pm 0,03$ & 4,63 \\
\hline & marrom & $3,65 \pm 0,07$ & $2,15 \pm 0,02$ & & & & $2,02 \pm 0,02$ & $1,82 \pm 0,02$ & & 9,64 \\
\hline & amarelo & $7,2 \pm 0,3$ & & & & & & & & 7,19 \\
\hline & vermelho & $2,84 \pm 0,04$ & $12,3 \pm 0,8$ & & & & $0,57 \pm 0,00$ & & & 15,67 \\
\hline \multirow[t]{6}{*}{$\mathrm{E}$} & rosa & & & & & traços & traços & traços & & \\
\hline & verde & $6,1 \pm 0,2$ & & & & & & & traços & 6,07 \\
\hline & amarelo & $6,0 \pm 0,2$ & traços & & & & & & & 5,97 \\
\hline & laranja & $1,58 \pm 0,01$ & $2,96 \pm 0,05$ & & & & & & & 4,54 \\
\hline & marrom & $2,48 \pm 0,03$ & & & & & $1,64 \pm 0,01$ & & traços & 4,12 \\
\hline & azul & & & & & & & & $0,45 \pm 0,00$ & 0,45 \\
\hline
\end{tabular}

*média e estimativas do desvio padrão $(n=4)$

Tais dados só confirmam o observado quando comparados visualmente à coloração de produtos similares nacionais e importados, principalmente da Argentina e Estados Unidos. Em geral, os produtos importados (marcas E e G) apresentam uma coloração mais intensa. Esta, talvez, fosse a justificativa de quatro corantes em todos da marca G. Embora as marcas E e G sejam produtos importados, para sua comercialização no país, deveriam seguir as normas vigentes no país. As gomas da marca $\mathrm{F}$ são produtos nacionais e também estão fora das especificações. Ainda em relação às gomas de mascar, em duas amostras da marca D a extração não foi completa, mesmo após várias tentativas com outros solventes e combinações de solventes para a extração, talvez pelo tipo de goma utilizada que mantém o corante retido em sua estrutura, no entanto, consideramos que o mesmo ocorre durante o consumo.

O corante amaranto foi o único não encontrado nas amostras de cereais matinais coloridos (Tabela 3). As três marcas analisadas estão fora do permitido, sendo que na porção média recomendada pelos fabricantes o teor de corantes chegou a duas vezes mais que o permitido. A amostra C, além de apresentar altas concentrações de corantes artificiais, declarava na sua embalagem a presença de corantes artificiais, no entanto, as análises indicam não só a presença destes, mas também altas concentrações, superiores às permitidas pela legislação brasileira.

Nas amostras de balas apenas uma marca $(\mathrm{H})$ apresentou índices próximos ao máximo permitido, todas as demais marcas se apresentaram dentro da legislação (Tabela 4).

No geral, a tartrazina e o amarelo crepúsculo foram os corantes, quantitativamente, mais utilizados pelas indústrias nas amostras aqui analisadas.

Os cromatogramas referentes às amostras estão mostrados nas Figuras 1 e 2.
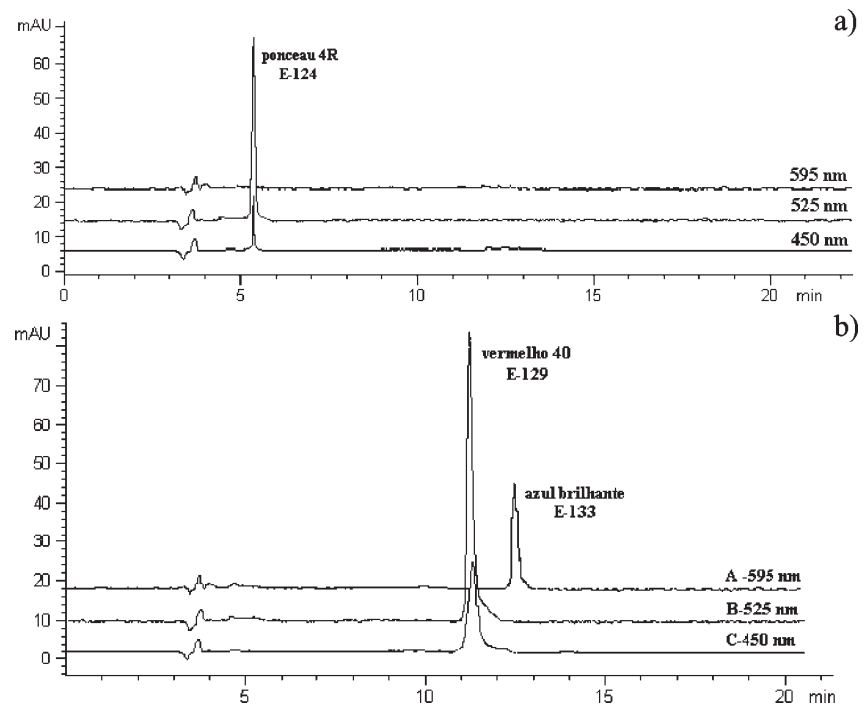

Figura 1. Perfis cromatográficos nos três comprimentos de onda de leitura para: (a) bala cor/sabor vermelha; (b) cereal matinal colorido cor/sabor roxa. Condições cromatográficas descritas no texto 
Tabela 2. Teores de corantes artificiais encontrados em gomas de mascar*

\begin{tabular}{|c|c|c|c|c|c|c|c|c|c|}
\hline MARCA & COR/SABOR & E-102 & E-110 & $\begin{array}{c}\text { CORANTES } \\
\text { E-123 }\end{array}$ & $\begin{array}{c}(\mathrm{mg} / 100 \mathrm{~g}) \\
\mathrm{E}-127\end{array}$ & E-129 & E-132 & E-133 & TOTAL \\
\hline \multirow[t]{4}{*}{ A } & laranja & & $27 \pm 1$ & & & $0,22 \pm 0,01$ & & & 27,32 \\
\hline & limão & $155 \pm 7$ & & & & & & & 154,77 \\
\hline & maçã & $0,26 \pm 0,01$ & & & & $13 \pm 1$ & & & 12,99 \\
\hline & melancia & $4,9 \pm 0,3$ & & & & $0,41 \pm 0,02$ & & & 5,34 \\
\hline \multirow[t]{5}{*}{$\mathrm{B}$} & verde & $6,5 \pm 0,3$ & & & & $0,17 \pm 0,01$ & & $2,04 \pm 0,06$ & 8,70 \\
\hline & laranja & & $12,8 \pm 0,6$ & & & & & & 12,82 \\
\hline & amarelo & $7,6 \pm 0,4$ & & & & & & & 7,56 \\
\hline & vermelho & & & & & $24 \pm 1$ & & & 23,45 \\
\hline & rosa & & & & & $7,3 \pm 0,4$ & & & 7,26 \\
\hline \multirow[t]{4}{*}{$\mathrm{C}$} & tutti frutti & & & & $2,6 \pm 0,1$ & $0,97 \pm 0,05$ & & & 3,57 \\
\hline & hortelã & $3,6 \pm 0,2$ & & & & $0,38 \pm 0,02$ & $1,04 \pm 0,05$ & & 5,05 \\
\hline & uva & & & $2,00 \pm 0,1$ & & $0,53 \pm 0,03$ & $0,74 \pm 0,04$ & & 3,27 \\
\hline & morango & & & & & $2,4 \pm 0,1$ & & & 2,41 \\
\hline \multirow[t]{4}{*}{$\mathrm{D}$} & uva & & & & & $2,5 \pm 0,1$ & $0,37 \pm 0,02$ & & 2,83 \\
\hline & cereja & & & & & $50 \pm 3$ & & & 50,25 \\
\hline & tutti frutti & & & & $1,95 \pm 0,09$ & $0,45 \pm 0,02$ & & & 2,40 \\
\hline & canela & & $34 \pm 2$ & & & $39 \pm 2$ & & & 72,95 \\
\hline $\mathrm{E}$ & pink & $7,5 \pm 0,4$ & $3,7 \pm 0,2$ & & & $33 \pm 2$ & & & 44,07 \\
\hline \multirow[t]{6}{*}{$\mathrm{F}$} & verde & $36 \pm 2$ & & & & $0,41 \pm 0,02$ & $14,2 \pm 0,7$ & & 50,12 \\
\hline & amarelo & $21 \pm 1$ & & & & & & & 21,37 \\
\hline & laranja & $55 \pm 3$ & & & $13,9 \pm 0,7$ & & & & 69,01 \\
\hline & rosa & $1,19 \pm 0,06$ & & & $26 \pm 1$ & $0,48 \pm 0,02$ & & & 27,78 \\
\hline & roxo & & & $29 \pm 1$ & $3,1 \pm 0,2$ & $1,28 \pm 0,06$ & & & 32,80 \\
\hline & bordo & & & & & $36 \pm 2$ & & & 36,16 \\
\hline \multirow[t]{3}{*}{ G } & azul/amarelo & $10 \pm 2$ & $1,09 \pm 0,00$ & & & $0,52 \pm 0,06$ & & $14 \pm 2$ & 25,59 \\
\hline & laranja/amarelo & $10,1 \pm 0,9$ & $8,9 \pm 0,4$ & & & $3,44 \pm 0,01$ & & $0,43 \pm 0,03$ & 22,84 \\
\hline & vermelho/laranja & $6,96 \pm 0,01$ & $9,43 \pm 0,00$ & & & $31,6 \pm 0,2$ & & $0,49 \pm 0,00$ & 48,49 \\
\hline
\end{tabular}

*média e estimativas do desvio padrão $(n=4)$

Tabela 3. Teores dos corantes artificiais encontrados em cereais matinais*

\begin{tabular}{|c|c|c|c|c|c|c|c|c|c|}
\hline MARCA & COR/SABOR & E-102 & E-110 & $\begin{array}{c}\text { CORANTES } \\
\text { E-124 }\end{array}$ & E-127 & $\begin{array}{c}(\mathrm{mg} / 100 \mathrm{~g}) \\
\mathrm{E}-129\end{array}$ & E-132 & E-133 & TOTAL \\
\hline \multirow{5}{*}{ A } & verde & & $0,37 \pm 0,07$ & & & $0,23 \pm 0,05$ & & $2,59 \pm 0,9$ & 3,2 \\
\hline & laranja & & $46 \pm 1$ & & & $1,6 \pm 0,5$ & & & 47,7 \\
\hline & vermelha & & $5,2 \pm 0,4$ & & & $8,8 \pm 0,5$ & & & 14,0 \\
\hline & amarela & & $2,7 \pm 0,8$ & & & $0,55 \pm 0,03$ & & & 3,25 \\
\hline & roxa & & & & & $14,4 \pm 0,1$ & & $2,49 \pm 0,06$ & 16,89 \\
\hline \multirow{6}{*}{ B } & porção ** & & $12 \pm 2$ & & $0,09 \pm 0,09$ & $3,8 \pm 0,6$ & & $0,57 \pm 0,09$ & 15,96 \\
\hline & verde & $31 \pm 2$ & traços & & & traços & & $0,93 \pm 0,2$ & 31,97 \\
\hline & laranja & $1,1 \pm 0,2$ & $136 \pm 15$ & & & $136 \pm 15$ & & & 139,5 \\
\hline & vermelha & $0,6 \pm 0,2$ & $2,5 \pm 0,3$ & & & $2,5 \pm 0,3$ & & $0,43 \pm 0,06$ & 55,89 \\
\hline & amarela & $78 \pm 1$ & $2,29 \pm 0,05$ & & $0,2 \pm 0,2$ & $2,29 \pm 0,05$ & & & 80,57 \\
\hline & roxa & $1,1 \pm 0,2$ & & & & & & $9,8 \pm 0,2$ & 37,4 \\
\hline \multirow{6}{*}{$\mathrm{C}$} & porção ** & $26 \pm 3$ & $29, \pm 8$ & & & $29, \pm 8$ & & $1,4 \pm 0,2$ & 74,9 \\
\hline & verde & $45 \pm 2$ & $3,79 \pm 0,1$ & $0,95 \pm 0,00$ & & & $6,8 \pm 0,4$ & & 56,40 \\
\hline & laranja & $1,2 \pm 0,4$ & $123 \pm 4$ & & & & & & 124,34 \\
\hline & vermelho & & $7,6 \pm 0,2$ & $30 \pm 1$ & & & & & 37,46 \\
\hline & amarelo & $69,6 \pm 0,3$ & $4,4 \pm 0,2$ & & & & & & 74,06 \\
\hline & porção** & $23 \pm 2$ & $33 \pm 7$ & $5,8 \pm 0,6$ & & & $0,5 \pm 0,1$ & & 62,17 \\
\hline
\end{tabular}

\footnotetext{
* média e estimativa dos desvios padrões $(\mathrm{n}=4)$; ** porção recomendada pelo fabricante $(30 \mathrm{~g}),(\mathrm{n}=8)$
} 
Tabela 4. Teores de corantes artificiais encontrados em balas*

\begin{tabular}{|c|c|c|c|c|c|c|c|c|c|c|}
\hline \multirow[b]{2}{*}{ MARCA } & \multirow[b]{2}{*}{$\begin{array}{c}\text { COR/ } \\
\text { SABOR }\end{array}$} & \multirow[b]{2}{*}{ E-102 } & \multirow[b]{2}{*}{ E-110 } & \multicolumn{3}{|c|}{ CORANTES } & \multicolumn{2}{|l|}{$(\mathrm{mg} / 100 \mathrm{~g})$} & \multirow[b]{2}{*}{ E-133 } & \multirow[b]{2}{*}{ TOTAL } \\
\hline & & & & E-123 & E-124 & E-127 & E-129 & E-132 & & \\
\hline & morango & & & $0,67 \pm 0,00$ & & & & & & 0,67 \\
\hline \multirow[t]{2}{*}{ A } & $\begin{array}{l}\text { laranja } \\
\text { hortelã }\end{array}$ & & $1,64 \pm 0,01$ & & & & & & & 1,64 \\
\hline & morango & & & $2,79 \pm 0,04$ & & & & & & 2,79 \\
\hline \multirow[t]{3}{*}{ B } & tutti-frutti & & & $2,08 \pm 0,02$ & & & & & & 2,08 \\
\hline & abacaxi & $12,8 \pm 0,8$ & & traços & & & & & & 12,84 \\
\hline & vermelho & & & & $14 \pm 1$ & & & & & 13,86 \\
\hline \multirow[t]{4}{*}{$\mathrm{C}$} & laranja & $9,1 \pm 0,4$ & & traços & & $1,96 \pm 0,02$ & & & & 11,05 \\
\hline & rosa & $0,29 \pm 0,00$ & & & & $1,65 \pm 0,01$ & & & & 1,94 \\
\hline & laranja & & $14 \pm 1$ & & & & $0,49 \pm 0,00$ & & & 16,62 \\
\hline & verde & $15 \pm 1$ & & & & & & & $0,90 \pm 0,01$ & 15,80 \\
\hline \multirow[t]{4}{*}{$\mathrm{D}$} & branco & traços & & & & & & & & \\
\hline & vermelho & & & & & & $8,6 \pm 0,4$ & & & 8,62 \\
\hline & amarelo & $5,6 \pm 0,2$ & & & & & $0,23 \pm 0,00$ & & & 5,82 \\
\hline & verde/amarelo & $5,7 \pm 0,2$ & & & & & & & $0,74 \pm 0,00$ & 6,43 \\
\hline \multirow{3}{*}{$\mathrm{v}$} & vermelho/branco & & & & & & $4,8 \pm 0,1$ & & & 4,79 \\
\hline & vermelho/laranja & & $8,7 \pm 0,4$ & & & & $6,9 \pm 0,2$ & & & 15,56 \\
\hline & verde/amarelo & $6,7 \pm 0,2$ & & & & & & $0,60 \pm 0,00$ & & 7,25 \\
\hline \multirow[t]{3}{*}{$\mathrm{F}$} & laranja/amarelo & $4,9 \pm 0,1$ & $12,4 \pm 0,8$ & & & & & & & 17,27 \\
\hline & rosa/amarelo & $4,6 \pm 0,1$ & & & & & $3,67 \pm 0,07$ & & & 8,31 \\
\hline & azul/rosa & traços & & & & & $1,89 \pm 0,02$ & & $2,30 \pm 0,03$ & 4,19 \\
\hline \multirow[t]{4}{*}{ G } & rosa/amarelo & $8,8 \pm 0,4$ & & & & & $7,1 \pm 0,3$ & & & 15,92 \\
\hline & laranja/verde & $1,20 \pm 0,01$ & $7,3 \pm 0,3$ & & & & $0,35 \pm 0,00$ & & $0,45 \pm 0,03$ & 9,27 \\
\hline & verde/amarelo & $6,9 \pm 0,2$ & & & & & & & $0,43 \pm 0,00$ & 7,39 \\
\hline & laranja & $9,00 \pm 0,4$ & $16 \pm 1$ & & & & $0,17 \pm 0,00$ & & & 25,17 \\
\hline \multirow[t]{3}{*}{$\mathrm{H}$} & amarelo & $16 \pm 1$ & & & & & $0,18 \pm 0,00$ & & & 15,87 \\
\hline & verde & $3,17 \pm 0,05$ & & & & & & & $0,52 \pm 0,00$ & 3,69 \\
\hline & vermelho & & & & & & $11,0 \pm 0,6$ & & & 11,03 \\
\hline
\end{tabular}

*média e estimativas do desvio padrão $(n=4)$
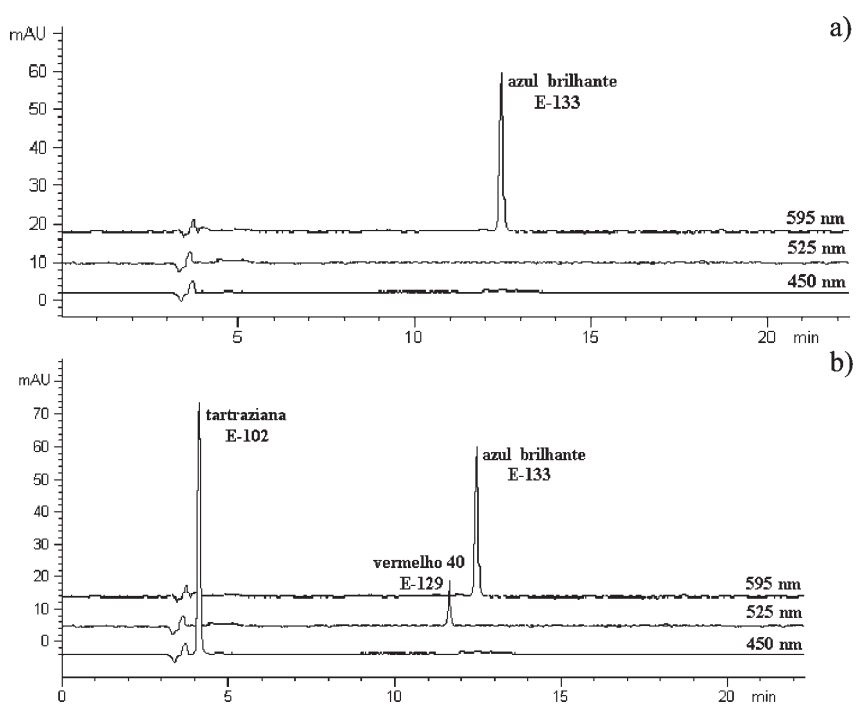

Figura 2. Perfis cromatográficos nos três comprimentos de onda de leitura para: (a) confeito de chocolate cor/sabor azul; (b) goma de mascar corl sabor verde. Condições cromatográficas descritas no texto

\section{CONCLUSÃO}

A metodologia empregada mostrou-se bastante eficaz para a quantificação de oito corantes artificiais permitidos no Brasil, nas amostras analisadas.

Os resultados apontam a não existência de um bom controle no uso de corantes artificiais pelas empresas alimentícias nacionais, já que muitos dos produtos analisados apresentavam teores acima dos permitidos pela legislação brasileira, que variam de 5 a $30 \mathrm{mg} / 100 \mathrm{~g}$. Os produtos importados deveriam atender às especificações da nossa legislação para serem comercializados no país.

Isto é um ponto preocupante, uma vez que esses produtos se destinam, principalmente, ao público infantil e o risco à saúde aumenta bastante neste tipo de consumidor. É necessária maior fiscalização por parte das autoridades para a liberação desses produtos, no que diz respeito aos teores de corantes artificiais utilizados pelas indústrias.

Embora não se tenha encontrado corantes não permitidos, as quantidades e misturas, muitas vezes, ultrapassaram os limites determinados. 


\section{REFERÊNCIAS}

1. GUIA OESP - Para a indústria de alimentação, painel do setor, 1991, p. 25

2. Prado, M. A.; Godoy, H. T.; Quim. Nova, 2004, 27, 22.

3. Prado, M. A.; Godoy, H. T.; J. Liq. Chromotogr. Relat. Technol. 2002, 25, 2455

4. Kapadia, J. G.; Tokuda, H.; Takayasu, J.; Ping, B.; Enjo, F.; Takasaki, M.; Konoshima, T.; Nishino, H.; Cancer Lett. 1998, 129, 87.

5. Rigs, J.; Brockow, K.; Behrendt, H.; J. Chromatogr., B: Anal. Technol. Biomed. Life Sci. 2001, 756, 3.
6. Ziena, H. M. S.; Youssef, M. M.; Aman, M. E.; Food Chem. 1997, 60, 501.

7. Tricard, C.; Cazabeil, J. M.; Medina, B.; Sci. Des Aliments 1998, 18, 25.

8. Reyes, F. G. R.; Prado, M. A.; Notícias ILSI 2001, 9, 4.

9. Prado, M. A.; Reyes, F. G. R.; Notícias ILSI 2003, 11, 3

10. http://www.anvisa.gov.br, acessada em Maio 2005.

11. Lancaster, F. E.; Lawrence, J. F.; Food Addit. Contam. 1991, 8, 249; PérezUrquiza, M.; Prat, M. D.; Beltrán, J. L.; J. Chromatogr., A 2000, 871, 227; Chen, Q.; Mou, S.; Hou, X.; Riviello, J. M.; NI, Z.; J. Chromatogr., A 1998, 827, 73; Gennaro, M. C.; Gioannini, E.; Angelino, S.; Aigotti, R.; Giacosa, D.; J. Chromatogr., A 1997, 767, 87; Horie H.; Kohata, K.; J Chromatogr., A 2000, 881, 425 . 